\title{
Die Rückkehr des Urbanen?
}

\author{
Christina West · Sebastian Lentz
}

Online publiziert: 29. März 2011

(C) Springer-Verlag 2011

Seit einiger Zeit sind in der Zeitschrift „Raumforschung und Raumordnung“ vermehrt Beiträge erschienen, die sich mit aktuellen Entwicklungen in Stadtregionen im Kontext der Fragestellung auseinandergesetzt haben, ob die Kernstädte nach Jahrzehnten des Bedeutungsverlusts wieder an Bedeutung gewinnen - eine Tendenz, für die oftmals der Begriff der „Reurbanisierung“ benutzt wird. Die in den raumwissenschaftlichen Publikationen zu diesem Thema behandelten Phänomene und die Forschungsperspektiven dazu sind allerdings sehr vielfältig, ja, sie erscheinen zum Teil sogar unübersichtlich: Zwar ist vom „Niedergang der Kernstädte" inzwischen kaum noch die Rede, aber Interpretationen von empirischen Befunden, bei denen anhand diverser Indikatoren auf eine Renaissance der Innenstädte oder gleich „des Städtischen“ überhaupt geschlossen werden soll, sind weit davon entfernt, paradigmatisch anerkannt zu sein. Für viele europäische Städte scheint zwar zu gelten, dass nach Jahrzehnten der Suburbanisierung die Bedeutung des Dekonzentrationstrends abnimmt; dies allerdings, ohne dass dafür ein einheitlicher Ursachenkomplex oder auch eine übergreifende Entwicklungsrichtung sichtbar werden: Die neue Unübersichtlichkeit hält - vorerst - an. Sie ist aber nicht zuletzt Folge einer allgemeinen Verbreiterung und Ausdifferenzierung der methodischen Zugänge in den Raumwissenschaften, die sich wiederum auf die Adaption und Weiterentwicklung theoretischer Konzepte sowie die Formulierung neuer Erkenntnisziele stützt.

C. West $(\bowtie)$

Lehrstuhl für Wirtschaftsgeographie, Universität Mannheim, L 7,

3-4, 68131 Mannheim, Deutschland

E-Mail: west@uni-mannheim.de

Prof. Dr. S. Lentz

Leibniz-Institut für Länderkunde, Schongauerstraße 9, 04329

Leipzig, Deutschland

E-Mail: s_lentz@ifl-leipzig.de
In grober Zusammenfassung mag man solche Arbeiten gruppieren, die einem siedlungsstrukturellen Paradigma verpflichtet sind, weshalb sie auf empirische Evidenz von quantitativ und räumlich dokumentierbaren (relativen) Konzentrationsprozessen in den inneren Bereichen von Stadtregionen zielen mit der Frage, ob sich damit neue Phasen der räumlich-strukturellen Entwicklung begründen lassen. Andere Beiträge zielen darauf ab, Erklärungen für die Ursache der neuen Attraktivität des „Städtischen“ aus Prozessen sozialer und ökonomischer Restrukturierung der Gesellschaft zu erarbeiten, wie z. B. der intergenerationellen Konstellation von Haushalts- und Familienformen unter dem Vorzeichen des demographischen Wandels oder den zunehmend transnational oder global verflochtenen Bedingungen der Erwerbsarbeit. Neuerdings erscheinen auch Arbeiten, die die neue Attraktivität des „Städtischen“ nicht nur entlang rationaler Faktoren oder mittlerweile vertraut erscheinender Konsummuster zu erklären versuchen. Sie widmen sich verstärkt Bedürfnissen, Einstellungen oder intrinsischen Motivationen für Entscheidungen; auch grundlegende Orientierungen etwa bei der Wohnstandortwahl mögen eine Rolle spielen. Regelmäßig setzen sie sich auch mit Interdependenzen zwischen Orientierungen auf gesellschaftlicher und individueller Ebene theoretisch auseinander und entwickeln neue Methoden zur empirischen Analyse. In einem weiten Sinne können all diese Arbeiten unter der Prämisse der Veränderung von Wertorientierungen zusammengefasst werden. Und schließlich gibt es Arbeiten, die ein sich wandelndes Verständnis urbanen Lebens und eine zumindest relativ gestiegene Attraktivität innerstädtischer Wohnquartiere in vielen Städten als gegeben annehmen, wofür die Aufwertung der Begriffe im semantischen Hof des „Urbanen“ im öffentlichen Diskurs ein ausreichendes Indiz sein mag. Daraus ergeben sich Ausgangspunkte für Fragen nach neuen Auseinandersetzungen um die Pro- 
duktion städtischer Räume sowie - weiter gefasst - um die (Neu-)Definition von Stadt und die Teilhabe an ihr in alltäglichen individuellen Praktiken ebenso wie jenseits persönlicher Lebensentwürfe.

In dieser Phase der theoretischen Unübersichtlichkeit haben wir auf dem Deutschen Geographentag 2009 in Wien eine Fachsitzung unter dem Titel „Die Rückkehr des Urbanen?" veranstaltet, um empirische Studien und theoretischkonzeptionelle Überlegungen zur Deutung der Phänomene und zur Diskussion dieser Frage zusammenzuführen. Mehr als 20 interessante Beitragsangebote erreichten uns, von denen wir schließlich acht Vorträge für eine Doppelveranstaltung berücksichtigen konnten. Die ersten dieser Vorträge werden nun in diesem Heft publiziert. Wir hoffen, dass weitere Arbeiten in künftigen Ausgaben von „Raumforschung und Raumordnung" folgen werden, die die Dynamik in dieser sowohl empirisch gestützten wie auch theoretisch inspirierten Diskussion um den Forschungsgegenstand „Stadt“ und sein Forschungsfeld zeigen. 